\title{
LES CORONAVIROSES AVIAIRES
}

\section{AVIAN CORONAVIROSES}

Par Jeanne BRUGERE-PICOUX'1 et Moncef BOUZOUAIA²

(Manuscrit reçu le 2 janvier 2021 et accepté le 20 avril 2021)

\section{RÉSUMÉ}

Les coronavirus sont des virus à ARN classés en Alphacoronavirus, Betacoronavirus, Gammacoronavirus et Deltacoronavirus. Ces virus semblent provenir des chauves-souris, et plus particulièrement les Alphacoronavirus et les Betacoronavirus (genre où l'on observe des zoonoses) alors que les oiseaux seraient à l'origine des Gammacoronavirus et des Deltacoronavirus. Le premier coronavirus identifié fut celui de la bronchite infectieuse aviaire en 1931 aux États-Unis. D'autres coronavirus ont été identifiés chez les oiseaux, proches ou différents du virus BI de la bronchite infectieuse aviaire et sont responsables de l'entérite de la dinde (TCoV) et de la maladie foudroyante de la pintade (GfCoV). Enfin, un deltacoronavirus peut être rencontré à la fois chez le porc (PD CoV UKU15) et les oiseaux.

Mots-clés : Coronavirus, Bronchite infectieuse aviaire, entérite de la dinde, syndrome entéritique mortel du dindonneau, maladie foudroyante de la pintade.

\begin{abstract}
Coronaviruses are RNA viruses classified into Alphacoronavirus, Betacoronavirus, Gammacoronavirus and Deltacoronavirus. These viruses seem to come from bats, and more particularly Alphacoronaviruses and Betacoronaviruses (the genus where zoonoses are observed), while birds are the source of Gammacoronaviruses and Deltacoronaviruses. The first coronavirus identified was that of avian infectious bronchitis in 1931 in the United States. Other coronaviruses have been identified in birds, similar to or different from the IB virus of avian infectious bronchitis and are responsible for turkey enteritis (TCOV) and fulminating guinea fowl disease (GfCoV). Finally, a deltacoronavirus can be found in both pigs (PD CoV UKU15) and birds (PRCV).

Key words : coronavirus, birds, avian infectious bronchitis, turkey enteritidis, Poult enteritis mortality syndrome, fulminating disease of guinea fowl
\end{abstract}

\section{INTRODUCTION}

La bronchite infectieuse $(\mathrm{BI})$ des volailles fut la première coronavirose décrite en 1931 par deus vétérinaires américains, Schalk et Hawn. Très rapidement on sut, toujours aux États-Unis, que l'agent était un virus filtrable (Beach et Schalm, 1936) qui fut identifié pour la première fois par son effet pathogène (nanisme et mortalité embryonnaire) après plusieurs passages sur œufs embryonnés par Beaudette et Hudson en 1937 d'où le nom de souche Beaudette donné à ce virus. Pendant longtemps, on a pensé que le virus BI n'affectait que l'espèce Gallus gallus, mais la gamme d'hôtes du virus BI s'est étendue à d'autres oiseaux comme la dinde (Meleagris gallopavo), le faisan (Phasianus colchicus), le paon (Pavo cristatus), la sarcelle (Anas), voire à un oiseau non gallinacé (Cavanagh, 2005, Liu, 2005). Cependant des coronavirus différents du virus BI ont été détectés chez des oies cendrées (Anser anser), des canards colvert (Anas platyrhynchos) et des pigeons (Columbia livia) (Cavanagh, 2005). Pendant un certain temps, un coronavirus du dindon (TCoV) fut considéré comme lié au coronavirus bovin qui est un Betacoronavirus mais des analyses ultérieures ont contredit cette hypothèse et démontré que ce TCoV était un Gammacoronavirus comme le virus BI tout en étant différent de ce dernier (Guy, 2000). Actuellement, les principales coronaviroses rencontrées chez les volailles sont la bronchite infectieuse aviaire, la coronavirose de la dinde et la maladie foudroyante de la pintade. Si ces principales coronaviroses des volailles sont dues

1. Académie nationale de médecine et Académie vétérinaire de France, Professeur honoraire de l'École nationale vétérinaire d'Alfort. Jeanne.brugere@orange.fr

2. Membre associé étranger Académie vétérinaire de France, Professeur École de médecine vétérinaire de Sidi-Thabet (Tunisie) 
à des gammacoronavirus, il importe de rappeler l'infection expérimentale de dindonneaux et de poulets par le deltacoronavirus PDCoV HKU de la diarrhée porcine à coronavirus (Boley et al. 2020) (Figure 1).

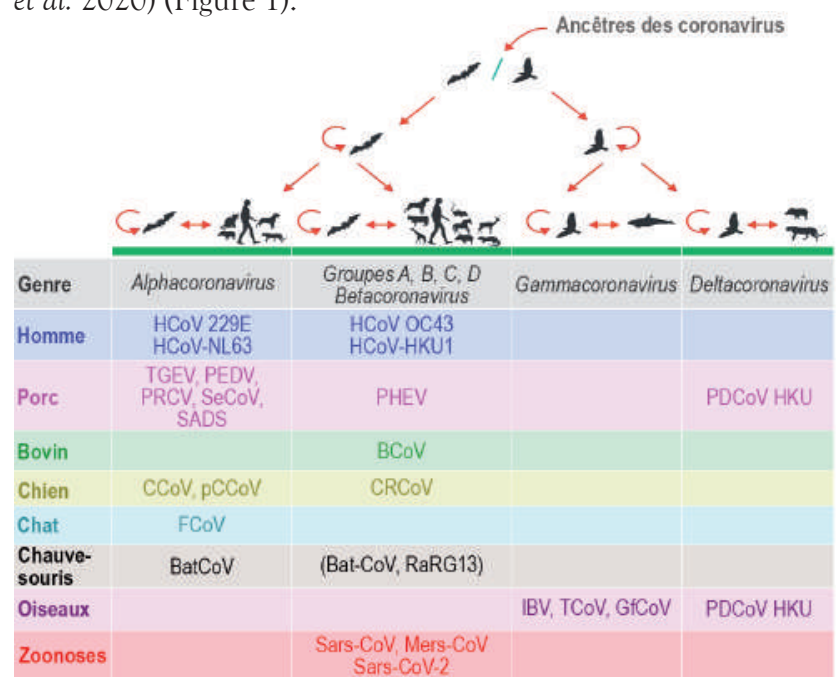

Figure 1 : Évolution des coronavirus, modifié d'après Woo et al. (2012) Parmi les Alphacoronavirus, certains peuvent contaminer l'Homme (HCoV 229E et HCoV-NL63) ou certains animaux domestiques : gastroentérite transmissible du porc (TGEV), diarrhée épidémique porcine (PEDV), coronavirose respiratoire du porc (PRCV), entérite porcine à coronavirus ( $\mathrm{SeCoV}$ ), diarrhée aiguë porcine (SADS), entérite à coronavirus $d u$ chien $(\mathrm{CCoV})$ ou pantropique (pCCo), coronavirose du chat (FCoV) présentant une forme entéritique (FECV) et une forme de péritonite (FIPV). Les Betacoronavirus peuvent être responsables d'une zoonose [syndrome respiratoire sévère ou SRAS (Sars-CoV), syndrome respiratoire du Moyen Orient ou MERS (Mers-CoV) et la Covid-19 (Sars-CoV2)] mais aussi de maladies respiratoires chez l'Homme (HCoV OC43 et HCoV-HKU1) ou d'une encéphalomyélite chez le porc (PHEV), de la coronavirose respiratoire du chien (CRCoV) ainsi que de la coronavirose bovine $(\mathrm{BCoV})$. On y retrouve aussi de nombreux coronavirus de la chauve-souris dont le virus Bat-CoV RaTG13 proche du Sars-CoV2. Chez les volailles on connaît principalement trois virus classés dans les Gammacoronavirus et correspondant à la bronchite infectieuse aviaire (IBV), la coronavirose du dindon (TCoV) et à celle de la pintade $\left(\mathrm{G} f V_{0} V\right)$, mais il faut remarquer la sensibilité des oiseaux au Deltacoronavirus de la diarrhée porcine PDCoV HKU.

\section{BRONCHITE INFECTIEUSE AVIAIRE}

La bronchite infectieuse aviaire (BIA) fut tout d'abord observée chez de jeunes poussins atteints d'une détresse respiratoire sévère. Dans les années 40, les essais de transmission expérimentale ont confirmé la grande contagiosité de cette maladie virale mais aussi la grande variété des lésions observées qui, outre les lésions respiratoires, concernent également les reins, l'oviducte avec des anomalies dans la formation de la coquille et de l'albumen. Malgré la variété du tableau clinique de cette maladie, le terme de «bronchite infectieuse " a été conservé. La BI représente une cause majeure de perte économique dans les élevages avicoles (ponte et chair) en particulier du fait de la chute de ponte très importante pouvant être observée sans un taux de mortalité important chez la poule adulte ou de l'atteinte respiratoire des jeunes. Elle est classée deuxième par la banque mondiale dans les maladies les plus préjudiciables à l'élevage avicole juste après l'influenza aviaire hautement pathogène (ou peste aviaire).

\section{Étiologie}

Cette maladie contagieuse se transmet par la voie aérienne et conjonctivale, le virus étant présent dans l'eau de boisson, la poussière et la litière. Puis le virus circule par contact entre les oiseaux mais on peut aussi le retrouver sur le matériel d'élevage (matériel d'emballage des oiseaux ou des œufs) ou sur des vecteurs animés comme le ténébrion (Alphitobius diaperinus) ou des araignées. Les souches sauvages du virus BI sont différenciées sur la base des symptômes et des lésions macroscopiques en pathotypes respiratoire, néphropathogène et entéritique. Les tests de neutralisation entre les sérums des oiseaux convalescents et diverses souches sauvages ont permis de dénombrer plusieurs sérotypes. Des souches vaccinales variées peuvent être groupées selon l'immunité conférée en protectotypes. L'analyse génomique de l'ARN peut permettre d'établir plusieurs génotypes. Dans les pays européens, les types du virus BI sont essentiellement du sérotype Massachusetts, les virus variants D274 et 1466, 793/B et B1648 ou la souche chinoise QX (Bouzouaia, 2021).

\section{Symptômes (Kaleta et Redmann, 2015)}

Les types et la sévérité des symptômes dépend de plusieurs facteurs (souche virale, résistance de l'hôte, poussières ou gaz délétères, infections secondaires bactériennes et/ou fungiques). Chez les jeunes poussins, il s'agit d'une maladie respiratoire (jetage séreux devenant purulent à la suite d'infections bactériennes secondaires). Des séquelles tardives peuvent être observées à l'âge adulte avec l'apparition de "fausses pondeuses", résultat de l'inflammation aiguë de l'épithélium de l'infundibulum. Chez les poulets et les poules reproductrices on observe un retard de croissance, une entérite et une néphrite. En dehors des atteintes respiratoires et rénales, les lésions de l'appareil génital sont caractéristiques chez les poules pondeuses et reproductrices. Les œufs pondus pendant la phase aiguë de la maladie contiennent un blanc d'œuf aqueux. La couleur, la grosseur et la solidité des œufs pondus varie énormément au sein du troupeau affecté (décoloration, fragilité, dépôts de calcium...) (figures 2 à 6).

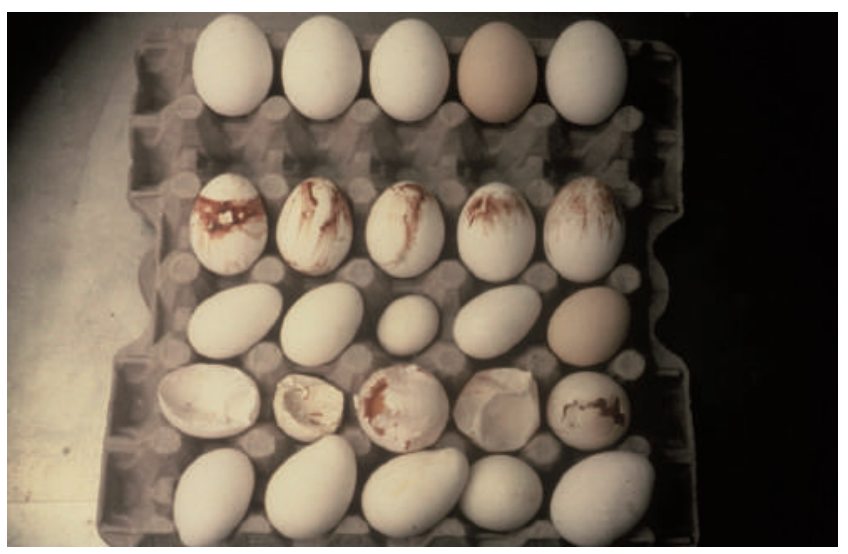

Figure 2 : Bronchite infectieuse. De haut en bas : œufs témoins, œufs tachés de sang, petits oufs, coquille d'œuf modifié (molle et se brisant facilement), œufs déformés (Photo J-P Picault). 


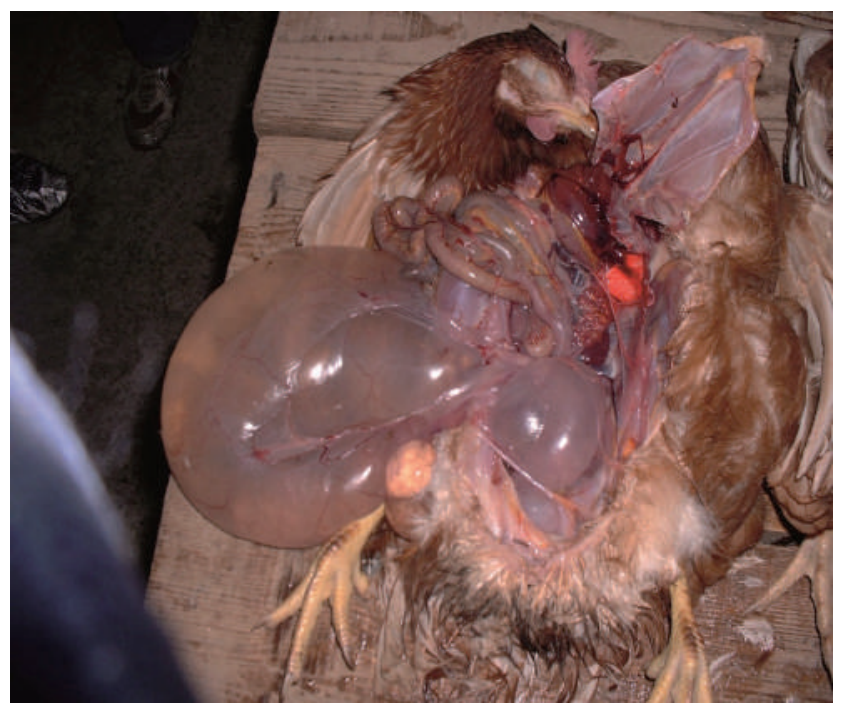

Figure 3 : Bronchite infectieuse. Présence d'un très gros kyste liquide dans l'oviducte d'une fausse pondeuse (Photo HJ Barnes).

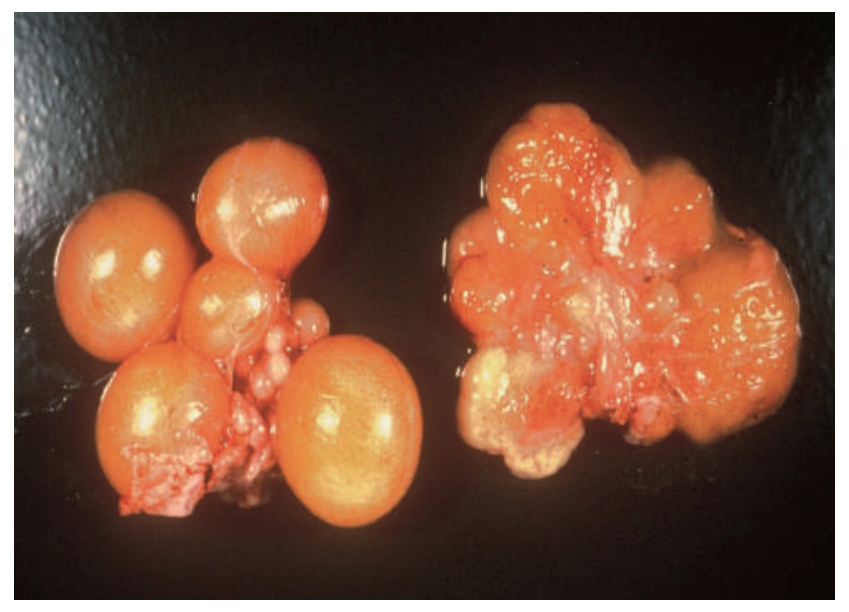

Figure 4 : Bronchite infectieuse. Comparaison entre un ovaire normal (à gauche) et un ovaire infecté (à droite) (Photo Sanders).

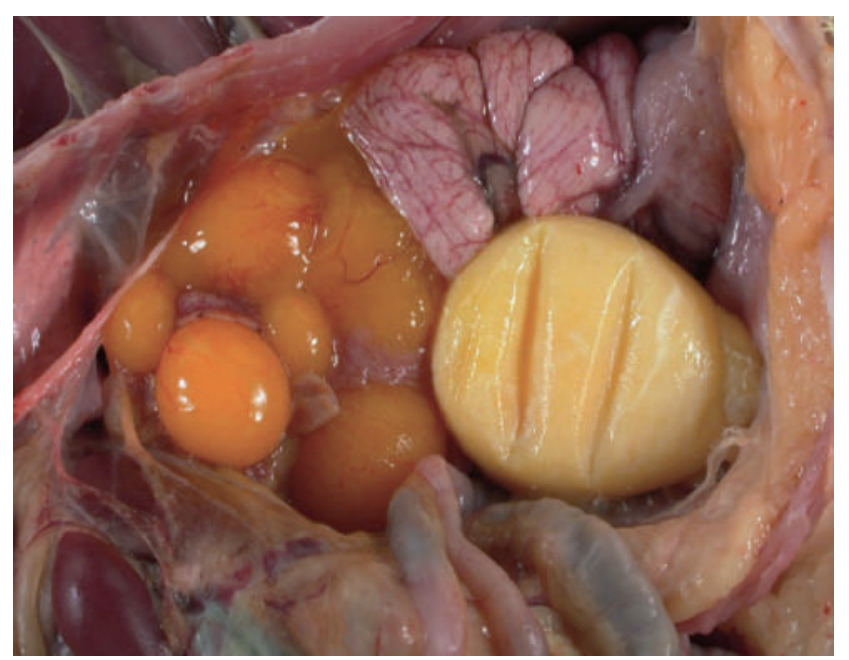

Figure 5: Bronchite infectieuse. Une ponte abdominale peut être observée chez les poules infectées (Photo HJ Barnes).

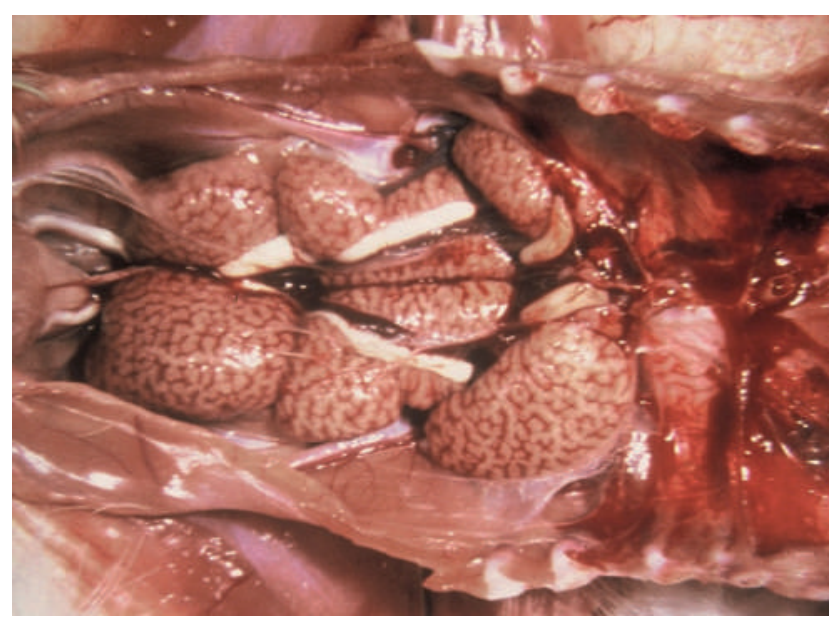

Figure 6 : Bronchite infectieuse. Néphrite sévère avec une importante hypertrophie rénale et une lithiase urinaire.

\section{Diagnostic}

Les symptômes et les lésions macroscopiques sont suggestives mais non pathognomoniques d'une atteinte par le virus BI. L'isolement du virus et sa caractérisation est d'une importance primordiale pour le diagnostic. Le virus BI fut l'un des premiers virus aviaires qui furent mis en culture sur les œufs embryonnés de poule. L'embryon de poussin demeure le premier choix pour l'isolement du virus BI. Les objectifs de cet isolement viral sont (i) la confirmation de la présence du virus BI, (ii) la détermination de son sérotype, et (iii) la détection d'autres virus aviaires concomitants. La technique très sensible de RT-PCR (reverse transcriptase polymerase chain reaction) est utilisée sur le virus BI cultivé sur œuf. Le séquençage des sondes PCR permet de différencier les souches vaccinales et les souches sauvages de même sérotype.

\section{Lutte contre la maladie}

En l'absence d'un traitement spécifique et du fait de la nature très contagieuse des virus BI, les seuls moyens de lutte sont la mise en place de mesures de biosécurité (veille épidémiologique, mesures de bioconfinement, nettoyage et vide sanitaire) et la vaccination. La vaccination nécessite au préalable une surveillance constante des génotypes circulant dans la région pour que les virus vaccinaux correspondent aux virus du terrain. Du fait de la grande variabilité du virus, le concept de protectotype a été développé par Cook et al. (1999) au laboratoire GD Deventer aux Pays-Bas. Un protectotype comprend l'ensemble des souches virales offrant la plus large protection contre les différents variants isolés sur le terrain permettant ainsi l'utilisation d'un seul vaccin. Aujourd'hui, tous les oiseaux d'élevage sont vaccinés et $80 \%$ d'entre eux, reçoivent une combinaison de vaccins de souche Mass et un vaccin préparé avec un virus variant, en application de la notion de protectotype.

CORONAVIROSE DU DINDON (Vaillancourt et al. 1997, 2015)

Le coronavirus du dindon (Turkey coronavirus ou TCoV) est responsable d'une entérite décrite pour la première fois en 1951 aux États-Unis (Peterson \& Hymas, 1951). Elle est caractérisée 
par une diarrhée, une dépression, une anorexie et une diminution du gain de poids. Avec le virus de l'entérite hémorragique de la dinde, le réovirus et l'astrovirus, il participe au complexe entérique du dindonneau (CED), ce terme englobant les maladies infectieuses intestinales des dindonneaux et incluant le syndrome entéritique mortel du dindonneau (SEMD) ou Poult enteritis mortality syndrome (PEMS). Le TCoV est considéré comme un agent causal du SEMD lorsqu'il est couplé avec d'autres agents pathogènes tels que certaines souches d'Escherichia coli (Guy \& Vaillancourt, 2015).

\section{Étiologie}

Le TCoV, isolé en 1971 (Adams \& Hofstad, 1971) n'affecte que la dinde et son rôle pathogène a été démontré en 1973. Le virus se réplique principalement dans les entérocytes du jéjunum et de l'iléon ainsi que dans l'épithélium de la bourse de Fabricius. Dans les intestins, la partie supérieure de la moitié aux deux tiers des villosités intestinales est la plus touchée. Le coronavirus du dindon est relativement résistant dans l'environnement. Il est stable à un $\mathrm{pH}$ de 3 à $22^{\circ} \mathrm{C}$ pendant 30 minutes et peut résister au moins une heure à $50^{\circ} \mathrm{C}$. Il peut survivre plus de cinq ans dans les tissus intestinaux conservés à $-20^{\circ} \mathrm{C}$ ou moins. Ceci explique pourquoi on a observé qu'il pouvait survivre sur le terrain pendant les longs hivers du Minnesota après l'élimination des dindes infectées. Le TCoV affecte les dindons pendant la période d'engraissement (après six semaines d'âge) mais la maladie clinique est surtout grave chez les oiseaux plus jeunes. Le TCoV est excrété pendant plusieurs semaines dans les fientes des oiseaux infectés, conduisant ainsi à une transmission horizontale par la litière utilisée, le matériel, les oiseaux vivants et morts ainsi que les vêtements et les chaussures contaminés du personnel. Les coléoptères et les mouches domestiques, les animaux nuisibles ou les chiens domestiques peuvent être des vecteurs mécaniques.

\section{Aspects cliniques}

Les symptômes surviennent généralement soudainement avec une morbidité élevée dans les troupeaux. On observe des retards de croissance et un taux de mortalité variable (surtout observé chez les dindonneaux). Chez les reproducteurs, une chute rapide de la production et de la qualité des œufs est notée. Cependant un troupeau peut être infecté sans présenter de signes cliniques évidents. Les lésions macroscopiques se trouvent principalement dans le tractus intestinal et la bourse de Fabricius. Le duodénum, le jéjunum et le cæcum sont remplis d'une matière liquide et gazeuse. La paroi intestinale est mince et flasque. De petites pétéchies peuvent être observées sur la muqueuse intestinale. Une atrophie de la bourse de Fabricius peut être notée. Lors d'une évolution chronique, les oiseaux seront amaigris et déshydratés (comme dans le SEMD). A l'examen microscopique, on note une diminution de la longueur des villosités, une augmentation de la profondeur des cryptes et une diminution du diamètre intestinal. Dans la bourse de Fabricius on observe une nécrose des cellules épithéliales.

Plusieurs tests de diagnostic sont disponibles, La technique de RT-PCR étant considérée comme un test de diagnostic très sensible et spécifique. L'entérite due au TCoV doit être différenciée des autres infections intestinales incluant celles associées aux astrovirus, aux rotavirus, aux réovirus, à Salmonella spp. et à Cryptosporidium spp.

\section{Méthodes de lutte}

Actuellement, les stratégies d'intervention comportent l'apport de médicaments et la gestion de la biosécurité. Un traitement de soutien est nécessaire dès le début d'apparition des signes cliniques. Il comporte l'apport de plusieurs vitamines solubles dans l'eau dont la vitamine $\mathrm{E}$ à deux fois la dose recommandée (en raison de ses propriétés anti-oxydantes qui aident à stabiliser les cellules épithéliales des villosités intestinales) ainsi qu'une antibiothérapie administrée dans l'eau de boisson lors de l'augmentation d'une mortalité liée à des co-infections. Une fois que la maladie est présente, l'antibiothérapie peut limiter la mortalité, mais n'empêchera pas la morbidité. Les soins palliatifs ne sont pas complets sans un effort soutenu visant à optimiser l'environnement. Une légère augmentation de la température ambiante $\left(1-2{ }^{\circ} \mathrm{C}\right)$ est souvent nécessaire car les oiseaux ont froid. Tous les efforts doivent être faits pour maintenir la litière aussi sèche que possible (en utilisant la ventilation, en retournant et/ou en rajoutant de la litière fraîche si nécessaire). Pour prévenir l'infection par le TCoV, une amélioration des mesures de biosécurité est essentielle, notamment en limitant les déplacements des personnes d'une ferme à l'autre (figures 7 à 11).

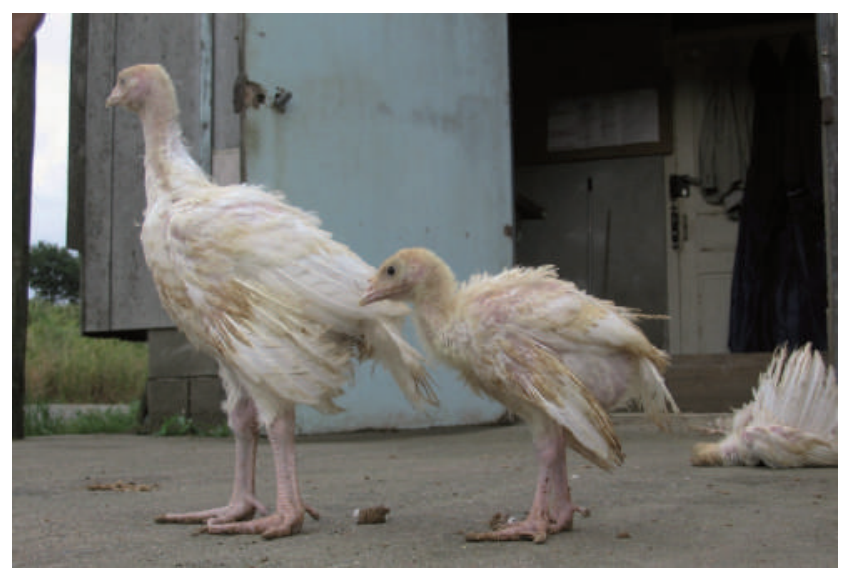

Figure 7 : Syndrome entéritique mortel du dindonneau. Le retard de croissance se traduisant par un troupeau non uniforme du troupeau va durer jusqu'à la fin de la production (Photo JY Ferré).

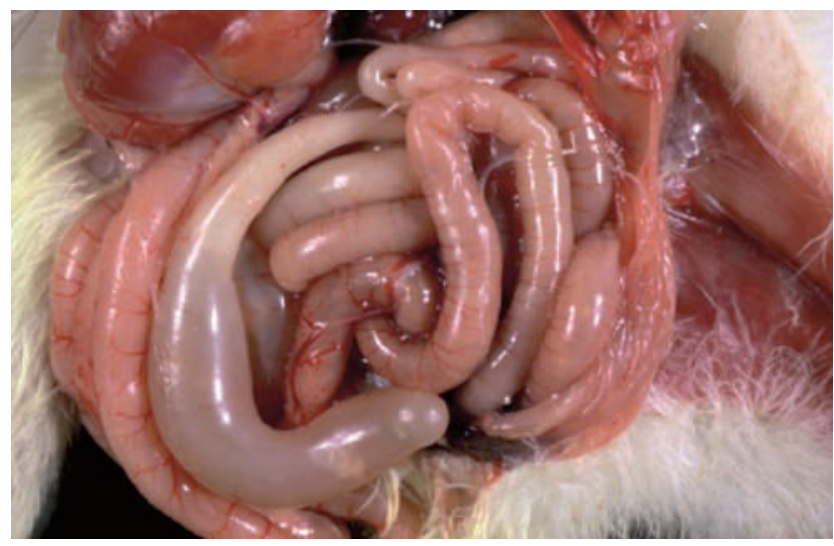

Figure 8 : Intestins d'un dindon atteint de Syndrome entéritique mortel du dindonneau. Remarquer l'amincissement et la pâleur de la paroi intestinale ainsi que la distension de l'intestin avec du liquide et du gaz (Photo JS Guy-AAAP). 


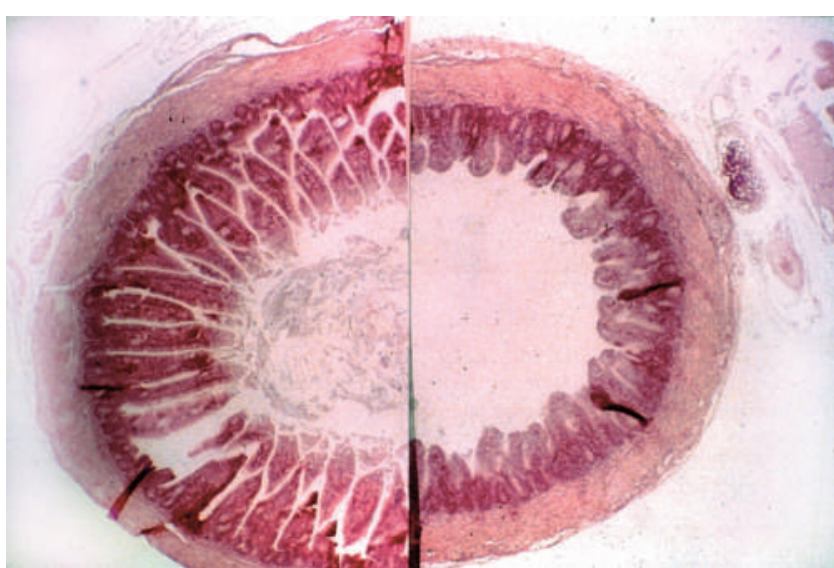

Figure 9 : Coronavirose du dindon. Au microscope, on observe une diminution de la longueur des villosités, une augmentation de la profondeur des cryptes et une diminution du diamètre intestinal. Comparer la longueur des villosités intestinales de l'intestin normal (à gauche) avec l'intestin affecté (à droite) (Photo HJ Barnes).

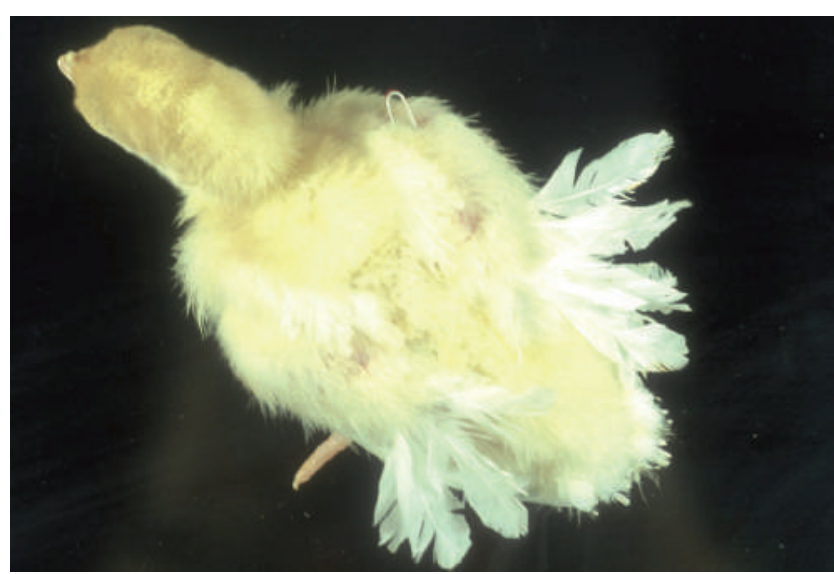

Figure 10 : Syndrome entéritique mortel du dindonneau. Le plumage des oiseaux survivants est anormalement fragile, leur donnant l'apparence d'un " hélicoptère " également décrit dans le syndrome " amaigrissement et retard de croissance " (Photo HJ Barnes).

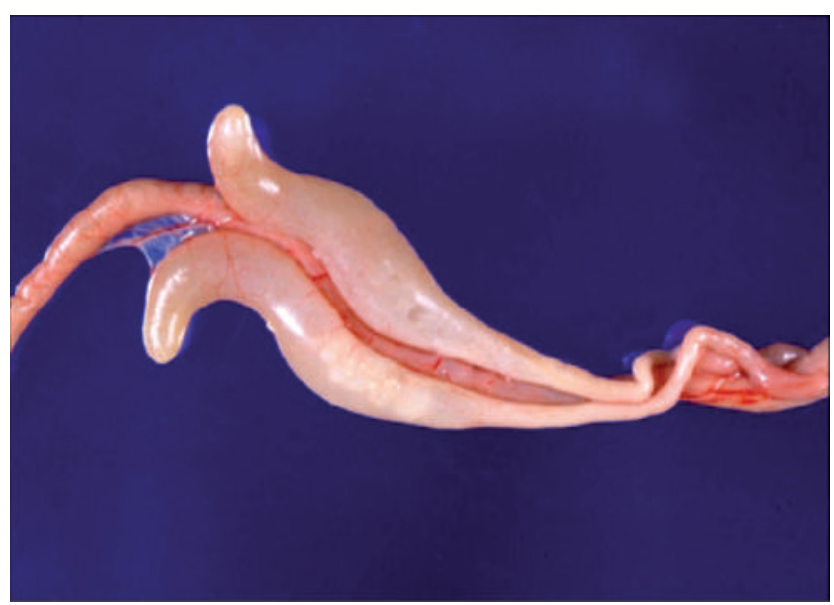

Figure 11 : Syndrome entéritique mortel $d u$ dindonneau. Inoculation expérimentale (7 jours PI). Cæcums nettement distendus par un contenu liquide brun pâle (Photo HJ Barnes).

\section{Syndrome entéritique mortel du dindonneau}

(Vaillancourt et al. 1997, 2015)

Le syndrome entéritique mortel du dindonneau (SEMD) ou Poult enteritis mortality syndrome (PEMS) a été d'abord décrit au début des années 90 dans le sud des États-Unis et est apparu beaucoup plus tardivement dans d'autres pays dont la France. Deux formes (Mortalité en pic du dindon et Excès de mortalité du dindon) ont été distinguées dans ce syndrome en fonction de leur gravité définie par le taux de mortalité. Dans ces deux formes, les dindonneaux sont atteints entre 7 et 28 jours d'âge. Actuellement le risque n'est plus limité au premier mois de vie mais couvre désormais l'ensemble de la période de démarrage (de $>1$ semaine jusqu'à 6 semaines). La Mortalité en pic du dindon (MPD) ou Spiking Mortality (SMT) se caractérise par une mortalité journalière supérieure ou égale à $1 \%$ par jour pendant au moins trois jours consécutifs ou par une mortalité journalière supérieure ou égale $9 \%$ pendant une période de trois semaines. L'Excès de mortalité du dindon (EMD) ou Excess mortality (EMT) se caractérise par une mortalité qui n'atteint pas ou ne dépasse pas $1 \%$ par jour pendant 3 jours consécutifs ou par une mortalité supérieure à $2 \%$ mais inférieure à $9 \%$ pendant une période de trois semaines. Il n'y a pas une étiologie simple associée à ce syndrome qui peut être contagieux. Il existe un consensus pour dire que la SEMD causé par plus d'un agent, probablement un virus, en association avec d'autres virus (par exemple, un coronavirus) et/ou des bactéries (par exemple Escherichia coli) et/ou des protozoaires (par exemple Cochlosoma, Spironucleus, Cryptosporidium).

Les symptômes caractérisant le SEMD sont la diarrhée, la déshydratation, la perte de poids, l'anorexie, le ralentissement de la croissance et la mort. Le retard de croissance dépasse souvent $40 \%$ et les survivants au SEMD ne présentent aucune croissance compensatrice. La maladie débute brusquement avec une morbidité proche de 100\%. Les survivants sont retirés avec des plumes ébouriffées (connus sous le nom d'oiseaux « hélicoptères " car beaucoup de plumes sont à des angles différents les unes des autres). Le taux de mortalité monte rapidement pour dépasser $1 \%$ /jour pendant plusieurs jours et peut dépasser $10 \% /$ jour dans les cas graves. Il n'y a pas de lésions pathognomoniques permettant le diagnostic du SEMD. Comme ce syndrome est vraisemblablement la conséquence de l'interaction de plus d'un agent infectieux, il n'existe aucun test de diagnostic formel disponible. Le diagnostic est basé sur l'évolution de la mortalité, l'absence d'une cause identifiable pour les signes cliniques observés. Certains agents connus parfois associés au syndrome peuvent être identifiés, comme le coronavirus de la dinde. Les moyens de lutte sont les mêmes que pour la coronavirose de la dinde.

\section{CORONAVIROSE DE LA PINTADE OU MALADIE FOUDROYANTE}

L'élevage de la pintade (Numida meleagris) est une particularité européenne, la France étant le principal producteur (figures 12 et 13). La maladie foudroyante ou maladie X est spécifique de la pintade et son étiologie virale (le GfCoV) a été démontrée par nos confrères de l'école nationale vétérinaire de Toulouse en 
2014 (Liais et al.). Elle apparaît brutalement à tout âge avec un taux de mortalité de 30 à $80 \%$ en 48 heures. L'autopsie révèle un contenu verdâtre liquide dans l'intestin, une distension des cæca avec un contenu jaune mousseux, une dilatation de la vésicule biliaire, une néphrite et une nécrose du pancréatique. Le diagnostic différentiel doit écarter l'hypothèse d'une peste aviaire due à virus influenza hautement pathogène (Roberton et al. 2015). Le coronavirus responsable GfCoV est proche de celui du TCoV.

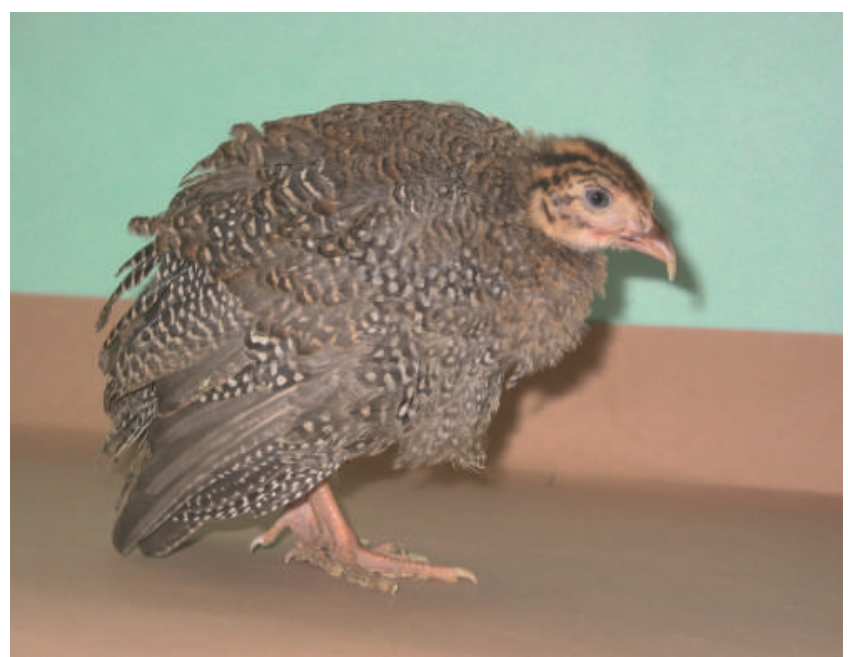

Figure 12 : Maladie foudroyante. Pintade prostrée âgée de 7 semaines (Photo JR Roberton).

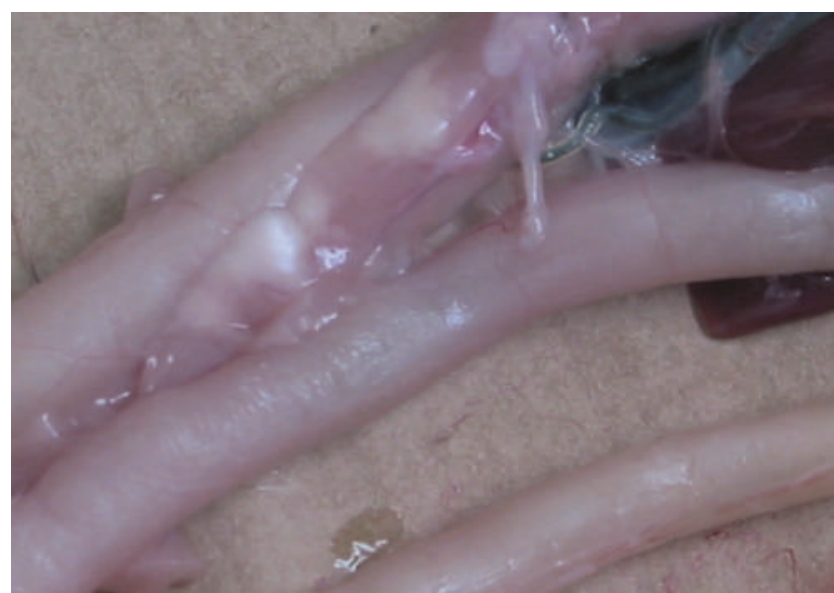

Figure 13 : Maladie foudroyante de la pintade. Aspect neigeux du pancréas (Photo JR Roberton).

\section{CONCLUSION}

Les coronaviroses aviaires sont les premiers coronavirus connus en médecine vétérinaire ou médicale et, comme les coronaviroses spécifiques aux espèces animales, elles ne sont pas zoonotiques. Elles représentent un excellent exemple des problèmes rencontrés actuellement dans la pandémie de la COVID-19, notamment quant aux problèmes rencontrés dans la lutte contre la bronchite infectieuse où les vaccins ont dû être adaptés à l'apparition de souches virales variantes.

\section{BIBLIOGRAPHIE}

- Adams NR, Hofstad MS. Isolation of transmissible enteritis agent of turkeys in avian embryos. Avian Dis 1971;15:426-433.

- Beach JR, Schalm OW. A filterable virus, distinct from that of laryngotracheitis, the cause of a respiratory disease of chick. Poultry Sci 1936;15:199-206.

Beaudette FR, Hudson CB. Cultivation of the virus of infectious bronchitis. J Am Vet Med Assoc 1937;90: 51-58.

- Boley PA, Alhamo MA, Lossie G, Yadav K, Vasquez-Lee M, Saif LJ, et al. Porcine Deltacoronavirus infection and transmission in poultry, United States. Emerg Infect Dis 2020;26:255-265. https://dx. doi.org/10.3201/eid2602.190346.

- Bouzouaia M. Les coronaviroses aviaires : caractéristiques présentant un intérêt épidémiologique en médecine comparée. Bull Acad Natl Méd, 2021, sous presse.

- Cavanagh D. Coronaviruses in poultry and other birds. Avian Pathol. 2005 Dec;34(6):439-48. doi: 10.1080/030794 50500367682. PMID: 16537157.

- Cook JK, Orbell SJ, Woods MA, Huggins $\mathrm{MB}$. Breadth of protection of the respira- tory tract provided by different live-attenuated infectious bronchitis vaccines against challenge with infectious bronchitis viruses of heterologous serotypes. Avian Pathol 1999;28: 477-85.

- Guy JS. Turkey coronavirus is more closely related to avian infectious bronchitis virus than to mammalian coronaviruses: a review. Avian Pathology (2000) 29, 207-212.

- Guy JS et Vaillancourt J-P. Coronavirus du dindon. Manuel de pathologie aviaire, Brugère-Picoux J, Vaillancourt J-P, Shivaprasad HL, Venne D et Bouzouaia M, Ed. AFAS, Paris 2015, p 243-247.

- Kaleta EF, Redmann T. Bronchite infectieuse. Manuel de pathologie aviaire, Brugère-Picoux J, Vaillancourt J-P, Shivaprasad HL, Venne D et Bouzouaia M, Ed. AFAS, Paris 2015, p 164-175.

- Liais E, Croville G, Mariette J, Delverdier M, Lucas MN, Klopp C et al. Novel avian coronavirus and fulminating disease in guinea fowl, France. Emerg Infect Dis 2014;20:105-8.

- Liu S, Chen J, Chen J, Kong X, Shao Y,
Han Z, Feng L, Cai X, Gu S, Liu M. Isolation of avian infectious bronchitis coronavirus from domestic peafowl (Pavo cristatus) and teal (Anas). J Gen Virol. 2005 Mar;86(Pt 3):719-725.

- Peterson EH, Hymas TA. Antibiotics in the treatment of unfamiliar turkey disease. Poutry Sci 1951;30:466-468.

- Roberton J, Robineau B, Doublet N. Élevage et maladies de la pintade. Manuel de pathologie aviaire, Brugère-Picoux J, Vaillancourt J-P, Shivaprasad HL, Venne D et Bouzouaia M, Ed. AFAS, Paris 2015, p 624-629.

- Schalk AF, Hawn MC. An Apparently New Respiratory Disease of Baby Chicks. J Am Vet Med Assoc 1931;78: 413-423.

- Vaillancourt JP, Barnes J, Guy J, Carver D, Wages D, Brugère- Picoux J. Syndrome entéritique mortel du dindonneau. Bull. Acad. Vét. de France 1997;70:243-250.

- Vaillancourt J-P, Carver D, Guy JS, Bartnes HJ. Syndrome entéritique mortel du dindonneau. Manuel de 
pathologie aviaire, Brugère-Picoux J, Vaillancourt J-P, Shivaprasad HL, Venne D et Bouzouaia M, Ed. AFAS, Paris 2015, p 484-491.

- Woo PC, Lau SK, Lam CS, Lau CC,
Tsang AK, Lau JH et al. Discovery of seven novel Mammalian and avian coronaviruses in the genus deltacoronavirus supports bat coronaviruses as the gene source of alphacoronavirus and betacoronavirus and avian coronaviruses as the gene source of gammacoronavirus and deltacoronavirus. J Virol. 2012; 86:3995-4008. doi: $10.1128 / \mathrm{JV}$ I.06540-11. 